\title{
The Magic of Buoyancy and Hydrostatics -Buoyancy and Effective Forces
}

\author{
Arnfinn Nergaard ${ }^{1}$, Naval Architect ${ }^{1} \&$ Petroleum Engineer ${ }^{1}$ \\ ${ }^{1}$ Department of Mechanical and Structural Engineering and Materials Science, University of Stavanger, Norway \\ Correspondence: Arnfinn Nergaard, Department of Mechanical and Structural Engineering and Materials \\ Science, University of Stavanger, Norway. E-mail: arnfinn.nergaard@uis.no
}

Received: October 16, 2017

Accepted: October 28, 2017

Online Published: November 28, 2017

doi:10.5539/mas.v11n12p77

URL: https://doi.org/10.5539/mas.v11n12p77

\begin{abstract}
More than 2000 years after Archimedes formulated his famous law, there is today still some disagreement as to the physical understanding and actual acceptance and application of Archimedes' Principle. This paper is prepared in the support of the full validity of Archimedes' Principle, always calculating buoyancy as the weight of displaced fluid, without exceptions.
\end{abstract}

Keywords: Archimedes, buoyancy, hydrostatics, effective forces

\section{Background}

Available literature on buoyancy effect in an oil well reveals that there are different ways of understanding the physics of buoyancy and hydrostatics when assessing forces in a drill pipe. Published articles and books reflect confusion in the industry with two different schools, one being the buoyancy school based on Buoyancy' law related to the effect of displaced fluid, the second being the pressure-on-a-flat or piston method school. The latter claims that there need to be an exposed flat subject to pressure from below to have buoyancy. Many text books in physics support this view explaining buoyancy with higher pressure at the bottom than at top of an immersed object. In a free floating situation both methods will yield the same gross upthrust, however, the problem emerges if an element has no vertical flat exposed to pressure from below, like a cylindrical, vertical riser connected to the seabed. The flat-supporters claim there is no buoyancy while volume-supporters stick to the principle of Archimedes giving the riser an upthrust equal to the weight of the displaced fluid.

Some authors go longer than just reflecting different understanding of the principle, some actually claim that Archimedes' Principle has shortcomings and does not always apply. As a matter-of-fact, there are views in which the critics express direct opposition to Archimedes' law!

In 1980 Goins, after having performed some simple experiments, concluded that; "buoyancy is equal to the weight of the displaced fluid is not always true" (Goins, 1981).

In 2014 F.M.S. Lima et al. stated: "It would not be surprising that Archimedes, one of the greatest geniuses of the ancient world, had enunciated his original propositions with remarkable precision and insight, however there are some instances which he did not realize. One of these instances is shown here to be an exception to Archimedes Principle" (F.M.S. Lima et. Al, 2014).

\section{Archimedes Principle}

History says that Archimedes formulated his famous law more than 2000 years ago:

"When a body is fully or partly immersed in a fluid, the buoyancy force is equal to the weight of the displaced fluid".

This is simple and square cut. Easy to understand and easy to apply. Discussion could have ended with that statement. However, at some point of time an alternative definition of the magnitude of the buoyancy force emerged:

"When a body is fully or partly immersed in a fluid, the buoyancy force is equal to the sum of external hydrostatic pressure forces acting on the object" 


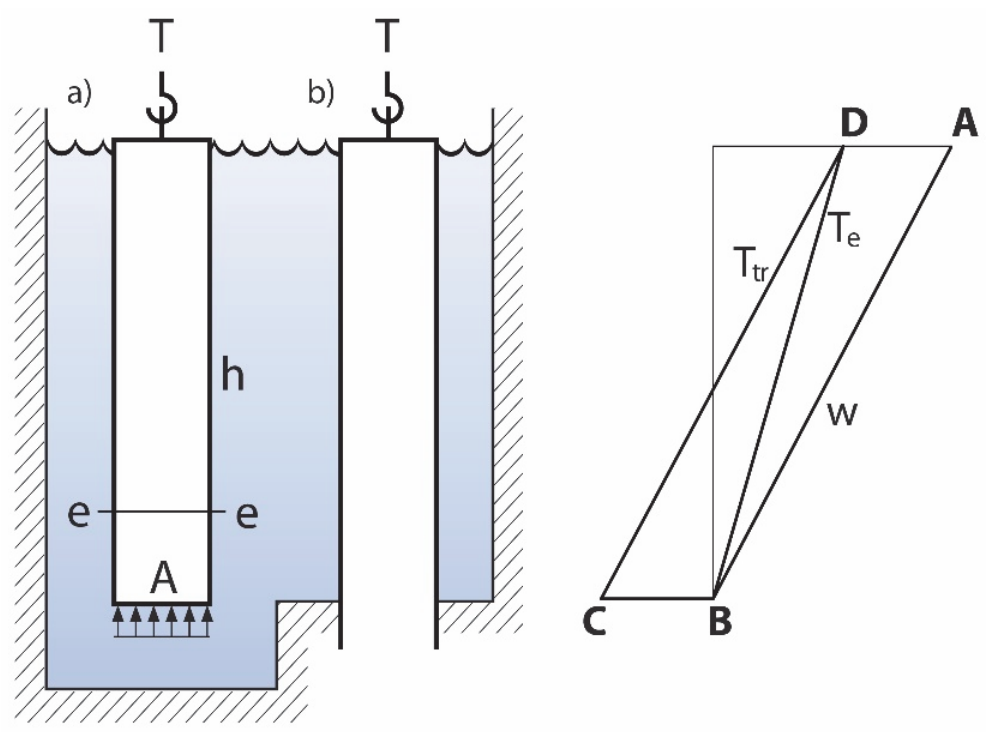

Figure 1. Immersion with and without flat

Fig 1 illustrates the two different ways to approach buoyancy. According to the above, case a) has buoyancy, case b) not. Firstly, considering case a), the object, a suspended bar (solid riser), has a dry tension illustrated by line AB. The pressure-on-flat school constructs the buoyancy as line $\mathrm{CB}(=\mathrm{pA})$ which is a point load at the bottom end resulting in a new axial force along the bar as line $\mathrm{CD}$, parallel to the dry weight line. This line is representing what literature refers to as true tension, $T_{\text {tr }}$

$$
\mathrm{T}_{\text {tr }}=\mathrm{w}(\mathrm{e})-\mathrm{p}_{\text {bottom }} \mathrm{A}
$$

where:

$\mathrm{T}_{\text {tr }}$ : True tension

$\mathrm{w}(\mathrm{e})$ : Weight of element below elevation e

$p_{\text {bottom }} \mathrm{A}$ : Hydrostatic force at bottom

However, the Archimedes' Principle (AP) gives a different axial tension line, at any level, as the weight minus the buoyancy of the element below the elevation, shown as line BD. This line is often referred to as the Archmedian line.

$$
\mathrm{T}_{\mathrm{a}}=\mathrm{w}(\mathrm{e})-\mathrm{B}(\mathrm{e})
$$

where:

$\mathrm{T}_{\mathrm{a}}$ : $\quad$ Archimedian tension

B(e): Buoyancy = weight of fluid displaced by element

C.P. Sparks introduced the term effective tension in 1984 and formulated the relation between true and effective tension (Sparks, 1984):

where:

$$
T_{e}=T_{t r}-p_{i} A_{i}+p_{e} A_{e}
$$

$\mathrm{T}_{\mathrm{e}}$ : $\quad$ Effective tension

$\mathrm{p}_{\mathrm{i}}, \mathrm{p}_{\mathrm{e}}$ : Pressure, internal, external

$\mathrm{A}_{\mathrm{i}}, \mathrm{A}_{\mathrm{e}}$ : Cross section area, internal, external

Combining equations (1) and (3) for the solid pipe above gives:

$$
\mathrm{T}_{\mathrm{e}}=\mathrm{w}(\mathrm{e})-\mathrm{p}_{\text {bottom }} \mathrm{A}+\mathrm{pA}=\mathrm{w}(\mathrm{e})-\mathrm{B}(\mathrm{e})
$$

This shows that Archimedian tension and the effective tension are identical as defined by line BD in Fig 1. Note that the effective tension at a certain level can be derived by using equation (2) directly, thus not needing to go via the true tension of equation (3). 
Now, given case b), with the riser connected at the bottom, and with the same top tension, the bottom hydrostatic force of case a) seem to be replaced by a mechanical true compression force from the bottom. According to AP both cases have the full effect of buoyancy while the AP critics claim that only case a) has buoyancy. While the critics claim that the buoyancy in this case appears at the bottom flat only (line CB) the AP implies that the buoyancy develops over the full volume of the element, represented by triangle $\mathrm{ABD}$, with full buoyancy seen only at the top, as line DA, identical for both cases.

Applying the above relations, and having the same top tension, the outcome is that cases b) and a) are equivalent mechanical systems implying that the force diagram shown represents both.

The following is concluded: A submerged riser pipe with a set top tension, with given properties and geometry is subject to the same true and effective tension independent of the pipe being connected to the bottom or not.

Both cases of Fig 1 have zero effective tension at the bottom. This assures that there is no buckling- or tension force. The true compression force at the bottom is a hydrostatic isotropic component not contributing to principal forces and hence not a part of the equation when dimensioning the riser for axial strength.

Now, consider a closed end pipe with fluid inside only, Fig. 2. The mechanical systems of Cases a) and b) are likewise found to be equivalent, however, as might be expected, with opposite effect as compared to the external fluid case.
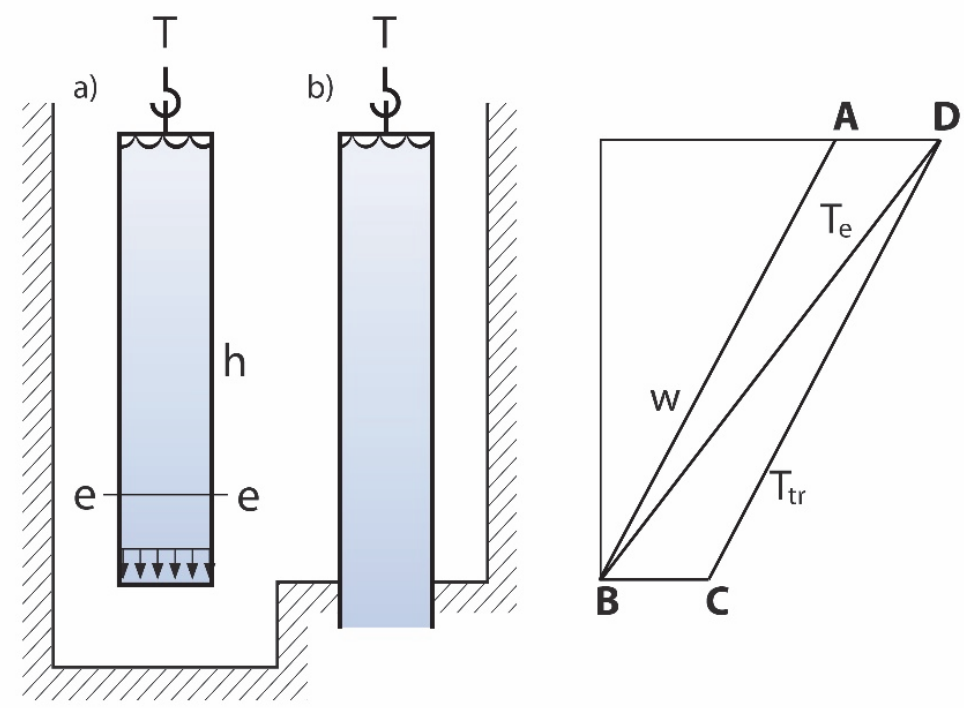

Figure 2. Fluid-filled pipe with and without 'bottom'

The effective tension is calculated from equation (3) as above. Alternatively, it can be found at any level directly as weight of pipe element below the level plus weight of fluid in the element. Again, the true tension (or compression) in terms of pressure on flats is not part of the equation when dimensioning the riser for axial strength. When true tension is considered the hydrostatic element must be deducted to get to the dimensioning tension, $\mathrm{T}_{\mathrm{e}}$, as demonstrated by equation 3 .

\section{Hydro-Mechanical Model for Effective Forces}

C.P. Sparks derived the relation between true- and effective tension and internal and external pressures in the very simple equation:

$$
\mathrm{T}_{\mathrm{e}}=\mathrm{T}_{\mathrm{tr}}-\mathrm{p}_{\mathrm{i}} \mathrm{A}_{\mathrm{i}}+\mathrm{p}_{\mathrm{e}} \mathrm{A}_{\mathrm{e}}
$$

The hydro-mechanical model and understanding that supports the equation is presented in his publications (Sparks 1984, 2005). The explanation is based on the acceptance of hydrostatic pressure 'invading' solid material and representing a force internally in the same manner as at the external flat. A simple illustration of this principle is presented in a force balance diagram in Fig 3. 


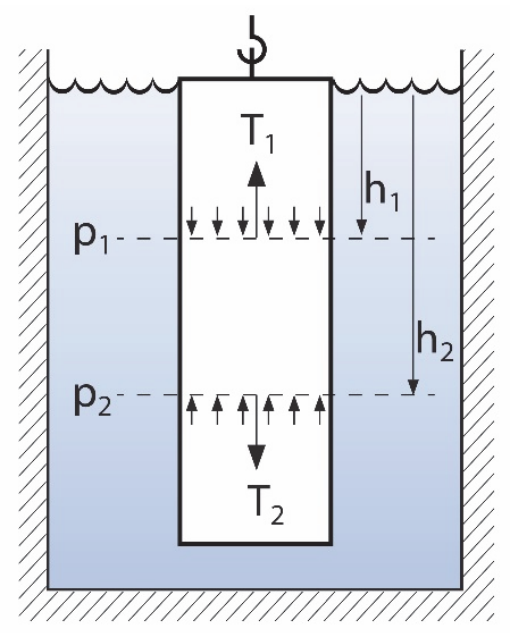

Figure 3. Force balance with internal hydrostatic forces

The figure shows an immersed solid pipe. A force balance between upward and downward working forces for the element between the dotted lines give:

$$
\mathrm{T}_{1}+\mathrm{p}_{2} \mathrm{~A}=\mathrm{p}_{1} \mathrm{~A}+\mathrm{w}+\mathrm{T}_{2}
$$

where:

$\mathrm{T}_{1}$ : Tension at top of element

$\mathrm{p}_{2}$ : Hydrostatic pressure at bottom

A: $\quad$ Cross section of pipe

$\mathrm{p}_{1}$ : Hydrostatic pressure at top

w: Weight of element

$\mathrm{T}_{2}$ : Tension at bottom element

This gives;

$$
\mathrm{T}_{1}-\mathrm{T}_{2}=\mathrm{w}-\left(\mathrm{p}_{2} \mathrm{~A}-\mathrm{p}_{1} \mathrm{~A}\right)=\mathrm{w}-\rho \mathrm{g}\left(\mathrm{h}_{2}-\mathrm{h}_{1}\right) \mathrm{A}=\mathrm{w}-\text { Weight of displaced fluid. }
$$

Starting at the bottom and moving up, this defines the effective tension equal to the Archimedian line, BD, in Fig 1. This shows that the Archimedian line not only coincides with effective tension, they are actually the very same line. This also shows that buoyancy is not depending on absolute pressure. Buoyancy is calculated on the basis of displaced fluid, however, pressure gradient may be used provided that internal hydrostatic pressures are included.

The principle shown in Fig 3, with hydrostatics invading a solid and having impact on vertical forces in the solid, is based on the 'assumption' that the hydrostatic pressure is isotropic, even inside solids. The fact that pressure in fluids is isotropic is fully and broadly accepted and understood. This is not the case for hydrostatic pressure/stress in solids.

The question is: How can it be that radial, horizontal pressure at the periphery of a vertical cylinder gives axial force inside?

In the following a demonstration of some effects supporting the fact that external peripheral pressure gives real axial forces are given:

- Analyzing riser for hydrostatic and deviatoric forces

- Von Mises equivalent stress from lateral pressure stress

- The Bridgman experiment

\section{Buoyancy and Deviatoric Forces}

For demonstrating the effect of external pressure on deviatoric forces in a vertical pipe, a demo example is shown in Fig 4. Imagine a solid cylinder with a starting stress (compression) from own weight equal to $\sigma$. In case a) the cylinder is exposed to external fluid only, in case b) to mechanical top tension T only. 


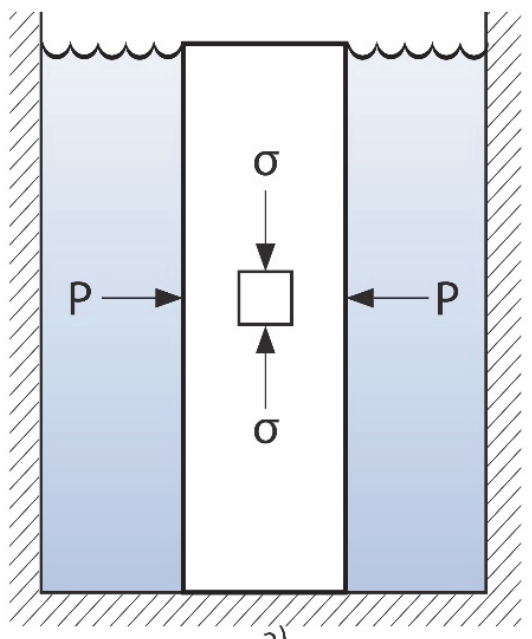

a)

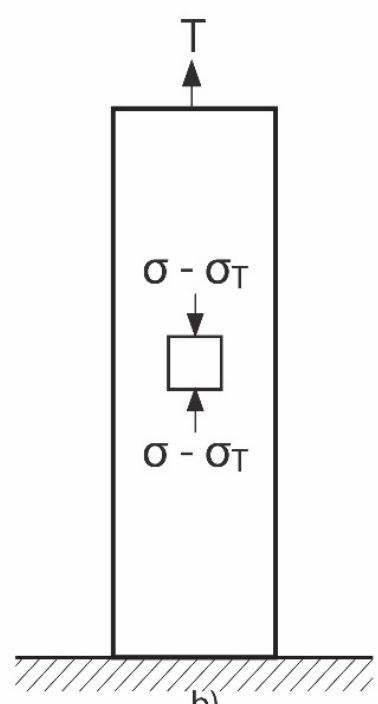

b)

Figure 4. Submerged vs dry riser

The objective of this demonstration is to derive the magnitude of the tension $\mathrm{T}$ to give a deviatoric tension at a chosen element in the riser equal to the deviatoric tension at the same level given only by external hydrostatic pressure for case a). The basic equations are:

$$
\begin{gathered}
\sigma_{\text {hydrostatic }}=\left(\sigma_{1}+\sigma_{2}+\sigma_{3}\right) / 3 \\
\sigma_{\text {total }}=\sigma_{\text {deviatoric }}+\sigma_{\text {hydrostatic }}
\end{gathered}
$$

Letting $\sigma_{1}$ represent axial - and $\sigma_{2}, \sigma_{3}$ lateral stress:

$$
\sigma_{1}=-\sigma, \sigma_{2}=\sigma_{3}=-p
$$

For Case a):

$$
\begin{gathered}
\sigma_{\text {hydrostatic }}=\left(\sigma_{1}+\sigma_{2}+\sigma_{3}\right) / 3=-(\sigma+2 p) / 3 \\
\sigma_{\text {deviatoric }}=\sigma_{\text {total }}-\sigma_{\text {hydrostatic }}=-\sigma+(\sigma+2 p) / 3=2 / 3(p-\sigma)
\end{gathered}
$$

For Case b):

$$
\begin{gathered}
\sigma_{\text {hydrostatic }}=\left(\sigma_{1}+\sigma_{2}+\sigma_{3}\right) / 3=-\left(\sigma-\sigma_{\mathrm{T}}\right) / 3 \\
\sigma_{\text {deviatoric }}=\sigma_{\text {total }}-\sigma_{\text {hydrostatic }}=-\left(\sigma-\sigma_{\mathrm{T}}\right)+\left(\sigma-\sigma_{\mathrm{T}}\right) / 3=2 / 3\left(\sigma_{\mathrm{T}}-\sigma\right)
\end{gathered}
$$

where

$\sigma_{\mathrm{T}}$ : Stress from force $\mathrm{T}$

Cases a) and b) have equivalent deviatoric tension stress for $\sigma_{T}=p=\rho g h$. This stress corresponds to a tension force $\mathrm{T}=\rho \mathrm{ghA}=$ Weight of the displaced fluid above the level considered.

This shows that axial deviatoric force resulting from lateral pressure at a given level is equal to the deviatoric force resulting from a mechanical tension force with a magnitude equal to the weight of the displaced fluid above the level.

\section{Buoyancy and von Mises Stress}

Consider case a) of Fig 4, a pipe exposed to lateral pressure only.

Letting $\sigma_{1}$ represent axial - and $\sigma_{2}, \sigma_{3}$ lateral stress:

$$
\sigma_{1}=-\sigma, \sigma_{2}=\sigma_{3}=-p
$$


Substituting these into the von Mises stress equation:

This gives the von Mises stress

$$
\sigma_{\mathrm{vM}}=\frac{1}{\sqrt{2}} \sqrt{\left(\sigma_{1}-\sigma_{2}\right)^{2}+\left(\sigma_{2}-\sigma_{3}\right)^{2}+\left(\sigma_{3}-\sigma_{1}\right)^{2}}
$$

$$
\sigma_{\mathrm{vM}}=\mathrm{p}-\sigma
$$

This means; the isolated effect of external fluid hydrostatic pressure on a vertical cylinder gives a von Mises equivalent or effective stress equal to the hydrostatic pressure. Over the cross section of the cylinder this stress corresponds to a force $\mathrm{T}=\rho \mathrm{ghA}=$ Weight of the displaced fluid above the level considered.

\section{Effect of Lateral Pressure - the Bridgman Experiment}

Bridgman's paradox:

In 1912, Percy Bridgman performed an experiment in which a cylindrical bar of glass was exposed to lateral pressure in a high-pressure test cell as shown in Fig 5 (Bridgman, 1912). The chamber was pressurized until the bar parted. Bridgman demonstrated how sufficiently high pressure in the chamber made the bar part as if in axial tension. This test specimen was clearly exposed only to radial pressure and hence, the surprising effect became known as Bridgman's paradox.

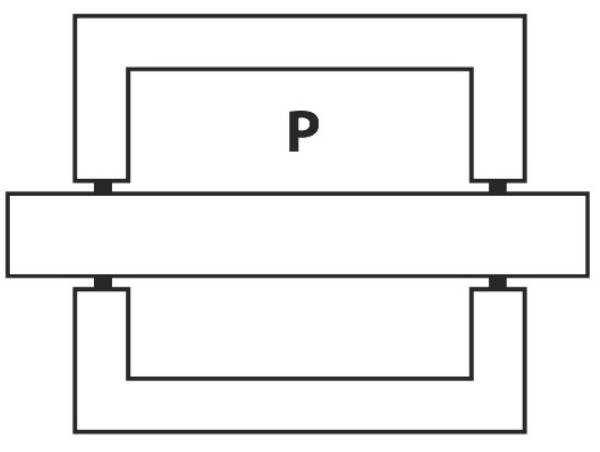

Figure 5. Bridgman test cell

Bridgman's experiment has been repeated in two Master projects at University of Stavanger, (Reve, 2013, Fossli, 2014). A cylindrical bar of $8 \mathrm{~mm}$ diameter acrylic thermoplastic material (PMMA) was exposed to increasing pressure until rupture. The PMMA material was specified to have a tensile strength range from 48 to $76 \mathrm{MPa}$. Reve found the bars to part at pressures from 67 to $75 \mathrm{MPa}$. The Bridgman test eliminates all other effects than external pressure in calculating the effective tension, thus equation (3) gives an effective axial tension:

$$
\mathrm{T}_{\mathrm{e}}=\mathrm{p}_{\mathrm{e}} \mathrm{A}_{\mathrm{e}}
$$

Over the cross section area, $A_{e}$, this corresponds to an effective stress $\sigma_{e}=p_{e}$. This means that external lateral pressure gives an axial tension stress equal to the lateral pressure. Part of the generated force is needed to overcome the friction in the sealing rings. Reve calculated the friction loss and concluded that the pressure contribution causing the rupture ranged from 60 to $68 \mathrm{MPa}$, a loss of about $10 \%$. This correlates well with the specified tensile strength range from 48 to $76 \mathrm{MPa}$.

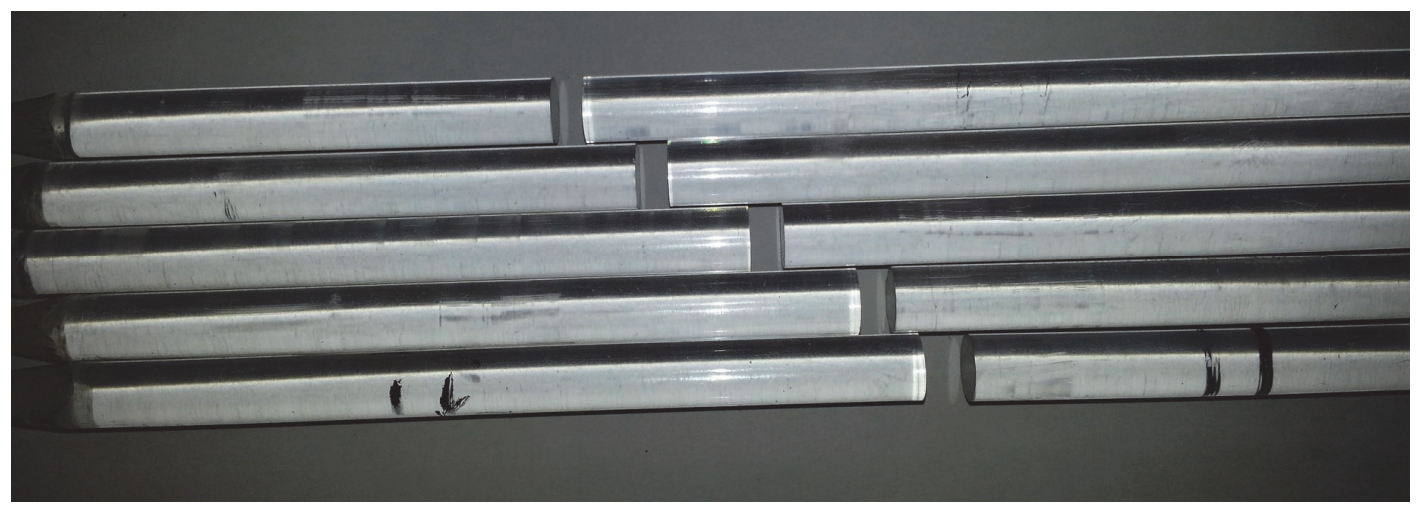

Figure 6. Parted acrylic bars ruptured by lateral pressure (Reve, 2013) 
Fig 6 shows five parted bars from one set of tests (Reve, 2013). The fracture seems to happen at different (random) locations between the glands of the test cell. Another important observation is that fracture surfaces are perfectly perpendicular to the longitudinal axis of the bar with a flat and shiny appearance. As a part of the project (Fossli, 2014) test specimens were also subject to bending fracture and to mechanical tensioning fracture in a tension test machine. In the bending experiment, the fracture surface had distinct tension and compression zones, the tension zone being perpendicular to the longitudinal axis of the rod.

In the tension machine experiment, the fracture surface was found to be perfectly perpendicular to the longitudinal axis of the rod as in the hydrostatic tests. Finally, the fracture surfaces were examined in a SEM (scanning electron microscope) with the following result; quote "The tension surface is similar to the fracture surfaces in both the Bridgman experiment and the tension side of the bending experiment".

\section{Conclusion}

This paper deals with the effect of radial pressure on axial forces in a cylindrical element. Effective tension theory says that external radial pressure on a cylinder gives an effective axial tension force equal to the pressure multiplied by the cylinder cross sectional area. This paper shows that effective tension in a vertical submerged cylinder corresponds with Archimedian tension. This is supported by demonstrating that lateral pressure at a given level gives axial tension at this level as if mechanically tensioned by a force equal to the weight of the displaced fluid above this level. Furthermore, Bridgman experiments at University of Stavanger have verified that the effective tension from lateral pressure tension the test specimen until fracture at a pressure equal to the tensile strength of the material. Finally, it is shown that applying effective tension for stress analysis is supported by classic analysis in the form of deviatoric stress theory and the von Mises criterion. Consequently, this implies that buoyancy develops over the volume of a submerged body and not from hydrostatic pressure on the bottom of the object.

This paper supports the full validity of Archimedes' principle, always calculating buoyancy as the weight of displaced fluid, without exceptions.

\section{References}

Goins, W. C. (1980). Better understanding prevents tubular buckling problems. Part 1- Buckling tendency, causes and resulting problems are described, World Oil, January 1980, pp. 101-105.

Kjetil, F. (2014). A study of fracture mechanisms when exposed to hydrostatic loads, Master Thesis, University of Stavanger.

Lima, F. M. S. et al (2014): A downward buoyant force experiment, Revista Brasileira de Ensino de Fisica, 36(2), 2309.

Morten, R. (2013). Understanding of Buoyancy in Drill Pipe and Risers, Master Thesis, University of Stavanger.

Sparks, C. P. (1984). The Influence of Tension, Pressure and Weight on Pipe and Riser Deformations and Stresses, Transaction of the ASME.

Sparks, C. P. (2005). Textbook: Fundamentals of Marine Riser Mechanics: Basic Principles and Simplified Analysis.

\section{Copyrights}

Copyright for this article is retained by the author(s), with first publication rights granted to the journal.

This is an open-access article distributed under the terms and conditions of the Creative Commons Attribution license (http://creativecommons.org/licenses/by/4.0/). 